\title{
The Effect of Ultra Low Concentrations of Some Biologically Active Substances on the Aerobic Respiration
}

\author{
Sergii V. Girin, Iryna V. Savinova, Iryna V. Antonenko, Natalia V. Naumenko \\ Cascade-Medical Reference Laboratory UBI, Glevakha, Ukraine \\ Email: girin@cascade-medical.com.ua
}

Received 17 February 2016; accepted 28 March 2016; published 31 March 2016

Copyright (C) 2016 by authors and Scientific Research Publishing Inc.

This work is licensed under the Creative Commons Attribution International License (CC BY). http://creativecommons.org/licenses/by/4.0/

(c) (i) Open Access

\begin{abstract}
For today it is known, that primary and secondary disorders of the aerobic respiration, which are based on mitochondrial deficiency, lead to a wide spectrum of clinical manifestations and diseases. Therefore, the question about effective correction of various types of energy exchange disorders remains topical. Thus, the aim of our work was the study effect of the complex of biologically active substances (BAS) in ultra low concentrations on the activity of key enzymes of aerobic energy metabolism succinate dehydrogenase (EC 1.3.99.1) (SQR) and mitochondrial glycerol-3-phosphate dehydrogenase (EC 1.1.99.5) (GPD2). The human lymphocytes assays were tested in vitro (22 donors). In negative control lymphocytes, cell culture normal saline solution was added. Normal saline solution with $\mathrm{NaN}_{3}$ was added in positive control lymphocytes cell culture. Experimental cell culture contained $\mathrm{NaN}_{3}$ and BAS. Our investigations had been revealed increase $S Q R$ activity in the experimental cell culture as compared with positive control culture throughout the time of experiment (measurements were carried out at $4,24,48,72 \mathrm{~h}$ of incubation). The $S Q R$ activity of experimental cell culture and negative control lymphocytes cell culture was equal up to $24 \mathrm{~h}$ of experiment. It showed neutralization of $\mathrm{NaN}_{3}$ inhibitory effect (during $24 \mathrm{~h}$ ) due to BAS influence. Activity of base glycerophosphate shunt ferment GPD2 of experimental lymphocyte cell culture was not different from GPD2 index in the negative control, but was lower than GPD2 activity in the positive control. It also indicated neutralization $\mathrm{NaN}_{3}$ inhibitory effect due to BAS influence. So we had found that extremely low concentrations of selected BAS with their complex impact on human lymphocytes in vitro could effectively neutralize the inhibitory effect of $\mathrm{NaN}_{3}$ on processes of aerobic energy metabolism link.
\end{abstract}

\section{Keywords}

Energy Metabolism, Ultralow Concentrations, Succinate Dehydrogenase, Glycerol-3-Phosphate Dehydrogenase 


\section{Introduction}

A key link of metabolism of any living system is an energy exchange. Both at the level of the whole organism, and at the level of an individual cell, the energy exchange is a grandiose complex process, which is regulated by multiplex and the subtlest manner and is organized in space and time.

Certainly the most important part of the energy metabolism is aerobic respiration. Due to this step energy exchange, organism receives most of the energy required for its life. Naturally, that practically any defects in the link of aerobic energy metabolism lead to significant structural and functional impairment, starting from the cellular level and ending with the whole organism. Currently, there are three type disorders of aerobic respiration: primary - a consequence of innate $\mathrm{nDNA}$ and mtDNA defects, disturbances caused by somatic mutations nDNA and mtDNA, and the so-called "energy-deficient diathesis" [1] [2].

For today it is known, that primary disorders of the aerobic respiration, which are based on mitochondrial deficiency, lead to a wide spectrum of nosological form. All of them first of all are dependent on violation aerobic respiration, as well as the degree of involvement in pathologic process of various tissues and organs. Mitochondrial medicine deals with issues of manifestation, prevention, epidemiology, diagnostic and therapy of mitochondrial diseases [3]-[6]. The clinical signs of mitochondrial failure are depend on type of aerobic respiration disturbance [2] [7]. Thus, disturbance of aerobic link of energy exchange in nervous tissue can be realized in a wide range of clinical signs from low tolerance to physical activity to severe encephalopathy, with the defeat of the cardiovascular system from moderate cardiac arrhythmia to cardiomyopathies. The violation of aerobic level of energy of nervous tissue can be implemented in a wide range of clinical signs low tolerance to physical load and to severe encephalopathy, with the defeat of the cardiovascular system from mild cardiac arrhythmia to cardiomyopathies. Aerobic respiration disorders, caused by somatic mutations in DNA and mtDNA, as well as "energy deficiency diathesis" have less serious consequences for the organism (secondary disorder). However, according to [7], the incidence of these disorders of aerobic energy exchange among children is about of $15 \%$ $20 \%$. It is important to note that with the age, this figure increases significantly and what its actual value is unknown. Thus, a significant part of the population has more or less pronounced energy deficit, and as a result, it needs effective and complex metabolic therapy, especially energotropic.

In the arsenal of doctors, there are several drugs able to activate specific inhibited reactions of energy exchange [8] [9]. However, some of them have low effectiveness while others have higher or lower toxicity. That's why it is not possible to use them for a long time. So the question about effective correction of various types of energy exchange disorders remains topical [10]. Research activities on homeopathy in recent years, at physical, chemical and biological levels with acceptable scientific norms and approach have paved the way for more rigorous research, particularly at the molecular level to understand the physicochemical nature and mechanism of action of ultra-high dilutions [11]-[13]. This is the main reason which leads to significant increase of preclinical and clinical trials of remedies which contain active substances in ultra low doses [14]-[16]. The perspective of this remedies' usage is determined by their safety, efficiency, better tolerability, almost total side effects absence and many other advantages [12] [16]. Unfortunately, nowadays of the number of remedies, providing influence on the energy metabolism and containing active substances in ultra low doses is insufficient. Moreover, their mechanism of action is still unclear. There is still an open question: how can such a small concentration cause changes in the intracellular energy exchange?

Therefore, the aim of our work was the study effect of the complex of biologically active substances (BAS) in ultra low concentrations on the activity of key enzymes of aerobic energy metabolism succinate dehydrogenase (SQR) and mitochondrial glycerol-3-phosphate dehydrogenase (GPD2).

\section{Experimental Part}

\subsection{Materials}

RPMI-1640 medium, Dulbecco's phosphate buffered saline (PBS), Histopaque-1077 Hybri-Max ${ }^{\circledR}$, Fetal Bovine Serum (FBS), L-glutamine, penicillin-streptomycin, $\alpha$-glycerol phosphate disodium salt hydrate, sodium succinate dibasic hexahydrate, potassium phosphate monobasic, sodium phosphate dibasic dihydrate and sodium hydroxide (Sigma-Aldrich Co., U.S.A.), ethylenediaminetetraacetic acid (EDTA) (Fluka Analytical, Switzerland), Janus green B, sodium azide, acetone and hydrochloric asid (Merck KGaA, Germany), trypan blue stain (Gibco ${ }^{\circledR}$ by Life Technologis, U.S.A.), p-nitrotetrazolium violet (RPC “Sinbias”, Ukraine), consumables for cell 
culture, cytochemistry, etc. (Sarstedt AG\&Co, Germany), tubes for blood sampling Venosafe ${ }^{\circledR}$ (Terumo Medical Corporation, Japan). Investigated complex of BASes (complex preparations Coenzyme Compositum ${ }^{\circledR}$, Ubichinon Compositum ${ }^{\circledR}$ ) in ultra low concentrations (“Heel”, Germany).

\subsection{Preparation of Primary Cultures of Human Lymphocytes}

The object of the study were initial human lymphocytes, which are obtained from the blood of 20 adults, 10 men and 10 women, aged 30 to 64 years, without obvious clinical symptoms of disease, serious chronic pathologies and harmful habits. Blood sampling was carried out from the cubital vein into tubes with anticoagulant EDTA (K2). Preparation of primary blood lymphocytes were performed using a sedimentation method [17] on the density gradient Histopaque-1077 Hybri-Max ${ }^{\circledR}$ (density $1.077 \pm 0.001 \mathrm{~g} / \mathrm{ml}$ ). Before layering the blood on a density gradient, it was diluted with sterile PBS without $\mathrm{Ca}^{2+}$ and $\mathrm{Mg}^{2+}$ of 1:1.25. Centrifugation was performed at $400 \mathrm{~g}$ for 30 min. The mononuclear fraction of blood was washed three times with sterile PBS without $\mathrm{Ca}^{2+}$ and $\mathrm{Mg}^{2+}$ The primary lymphocytes were separated from monocytes using adhesion of last on plastics. Mononuclear cells were incubated in a Petri dish for 30 minutes. The cells in the lymphocyte fraction were counted and evaluated viability in Goryaev chamber after trypan blue staining. Primary lymphocytes were cultured in PRMI-1640 which contained $100 \mathrm{ml}$ of 5\% FBS, $40 \mathrm{mM}$ L-glutamine, 10,000 Units penicillin and $10 \mathrm{mg}$ streptomycin. Cultivation was carried out in sterile Petri dishes at $37^{\circ} \mathrm{C}$ in an atmosphere which contained $5 \% \mathrm{CO}_{2}$ and $95 \%$ humidity.

\subsection{Preparation of Cell Culture $\mathrm{NaN}_{3}$ and BAS Treated}

Obtained pool of primary lymphocytes from one donor ( $1.5 \mathrm{ml}$ suspension) was divided into three equal portions of $0.5 \mathrm{ml}$ which were added to $12 \mathrm{ml}$ of the above medium and cultured as the negative control culture ( $\left.\mathrm{K}^{-}\right)$, positive control culture $\left(\mathrm{K}^{+}\right)$and experimental culture. At the expiration of the six-hour cultivation in the culture of $\mathrm{K}^{+}$and experimental culture of primary lymphocytes were added $0.5 \mathrm{ml}$ of $448 \mathrm{mM}$ solution of $\mathrm{NaN}_{3}$ in $\mathrm{PBS}$ without $\mathrm{Ca}^{2+}$ and $\mathrm{Mg}^{2+}$, and $0.5 \mathrm{ml}$ of PBS without $\mathrm{Ca}^{2+}$ and $\mathrm{Mg}^{2+}$ into culture $\mathrm{K}$. After 24 hours of culturing, from the moment of receiving primary lymphocytes, to the experimental culture of lymphocytes was added $1 \mathrm{ml}$ investigated complex of BAS diluted in a $0.9 \%$ solution of $\mathrm{NaCl}$. Simultaneously, to the cultures $\mathrm{K}^{+}$and $\mathrm{K}^{-}$was added $0.9 \mathrm{ml} 1 \%$ solution of $\mathrm{NaCl}$. The final concentration of BAS in the respective cultures media of primary lymphocytes was $3 \times 10^{6} / \mathrm{ml}, \mathrm{NaN}_{3} 16 \mathrm{mM}$ (Table 1). This concentration of BAS substances are ultralow [18]. As

Table 1. Content of the complex biologically active substances ${ }^{\mathrm{a}}$.

\begin{tabular}{|c|c|c|c|}
\hline Name of the substance & $\begin{array}{l}\text { The final concentration of } \\
\text { substances in the culture } \\
\text { medium }\left(10^{-12} \mathrm{~mol}\right)\end{array}$ & Name of the substance & $\begin{array}{l}\text { The final } \\
\text { concentration of } \\
\text { substances in the } \\
\text { culture medium } \\
\left(10^{-12} \text { mol }\right)\end{array}$ \\
\hline Coenzym A & 0.47 & Adenosintriphosphat-dinatrium & 0.01 \\
\hline Ascorbic acid & 223.11 & NAD & 0.55 \\
\hline Thiamine hydrochloride & 113.20 & Magnesium hydrogen phosphate & 236.88 \\
\hline Riboflavin sodium phosphate & 106.89 & Magnesium orotate dihydrate & 96.39 \\
\hline Pyridoxine hydrochloride & 191.18 & Cerous oxalate & 0.55 \\
\hline Nicotinamide & 321.75 & $\alpha$-Lipoic acid & 173.28 \\
\hline Cis-aconitic acid & 2.05 & Ubidecarenone & 0.01 \\
\hline Citric acid & 1.86 & Berberine & 40.43 \\
\hline Fumaric acid & 3.08 & Lactic acid & 396.43 \\
\hline$\alpha$-Ketoglutaric acid & 2.45 & Hydrochinon & 0.32 \\
\hline Malic acid & 2.66 & Hexaketocyclohexane octahydrate & 0.01 \\
\hline Succinic acid & 3.03 & Antraqinone & 0.01 \\
\hline Barium oxalsuccinate & 0.01 & Naphthoqinone & 0.02 \\
\hline Sodium diethyl oxalacetate & 170.06 & $p$-Benzoquinone & 0.03 \\
\hline Sodium pyruvate & 3.25 & Acetylsalicylic acid & 0.01 \\
\hline Cysteine & 294.68 & Histamine & 0.01 \\
\hline Sulfur copy & 11.25 & Magnesium D-gluconate-dihydrate and other & 0.01 \\
\hline
\end{tabular}

${ }^{\mathrm{a}}$ This complex of biologically active substances in composition corresponds to the drug Ubiquinone compositum ${ }^{\circledR}$ and Coenzyme compositum ${ }^{\circledR}$ (Biologische Heilmittel Heel GmbH, Baden-Baden, Germany). 
a result it was formed $\mathrm{K}^{-}, \mathrm{K}^{+}$and experimental cultures next compositions (Table 2).

Immediately before adding of the investigated complex BAS in experimental culture of primary cells and $0.9 \%$ $\mathrm{NaCl}$ solution to the control cultures and also after 4, 24, 48 and 72 hours after that, we obtained lymphocytes suspensions. Last were obtained by triple washing from the culture medium by PBS without $\mathrm{Ca}^{2+}$ and $\mathrm{Mg}^{2+}$ (centrifugation at $1200 \mathrm{~g}$ for 10 minutes.). In lymphocytes, using cytochemical methods of analysis, were determined the activity of key enzymes of energy metabolism-succinate dehydrogenase (SQR; EC 1.3.99.1) and mitochondrial glycerol-3-phosphate dehydrogenase (GPD2; EC 1.1.99.5) [19].

\subsection{Preparation for Cytochemical Investigations}

For carrying out cytochemical investigation we prepared smears of primary lymphocyte from suspensions obtained at different stages of the experiment. For this, in $3 \mu$ of primary lymphocytes suspension were added $2 \mu \mathrm{l}$ of authentic plasma. Received suspension mixture of primary lymphocytes and plasma authentic applied as small drops on a microscope slide. Touching upon drops, using cut glass and putting it at an angle of $45^{\circ}$, we made smears.

Fixation of smears preceded air drying of them over 2 hours to reduce the solubility of cellular components. Dried smears were fixed for 30 seconds in $60 \%$ aqueous acetone solution saturated with EDTA. 60\% aqueous solution of acetone saturated with EDTA were prepared by mixing $60 \mathrm{ml}$ of acetone and $40 \mathrm{ml}$ of water, then added EDTA to the termination its dissolvng. Fixed smears were washed with distilled water and dried in air at room temperature. Obtained smears were used to determine the activity of SQR and GPD2.

Incubation of lymphocytes to determine the succinate dehydrogenase. SQR activity was determined by cytochemical method. Smears of primary lymphocytes were incubated at $37^{\circ} \mathrm{C}$ for $1 \mathrm{~h}$ in the medium of the composition described in Table 3. If required the $\mathrm{pH}$ was adjusted with $0.1 \mathrm{~N}$ sodium hydroxide solution or $0.1 \mathrm{~N}$ hydrochloric acid solution to $\mathrm{pH}=7.3$. Received incubation medium was stored at $4^{\circ} \mathrm{C}$ not longer than 2 weeks. Incubation of lymphocytes for determining glycerol-3-phosphate dehydrogenase. As GPD2, SQR activity was determined by cytochemical method. Smears primary lymphocytes were incubated at $37^{\circ} \mathrm{C}$ for $1 \mathrm{~h}$ in the medium of the composition described in Table 4. The incubation medium had a $\mathrm{pH}=7.3$. If necessary, the $\mathrm{pH}$ was adjusted to the required value using $0.1 \mathrm{~N}$ sodium hydroxide solution or $0.1 \mathrm{~N}$ hydrochloric acid. Obtained incubation medium was stored at $4^{\circ} \mathrm{C}$ up to two weeks.

\subsection{Preparation, Micro Copying, Quantification of Succinate Dehydrogenase and Glycerol-3-Phosphate Dehydrogenase Activity}

All smears that were incubated for determining the activity of SQR, and for determining the activity of GPD2,

Table 2. Ingredients of primary lymphocyte cultures used in the experiment.

\begin{tabular}{|c|c|c|c|c|c|c|}
\hline Characteristics of the culture & $\begin{array}{c}\text { Medium of } \\
\text { cultivation } \\
\text { (RPMI-1640, FBS, } \\
\text { L-glutamine, } \\
\text { penicillin and } \\
\text { streptomycin), ml }\end{array}$ & $\begin{array}{l}\text { Suspension of } \\
\text { primary } \\
\text { lymphocyte } \\
\left(3 \times 10^{6}\right. \\
\text { cells } / \mathrm{ml}), \mathrm{ml}\end{array}$ & $\begin{array}{l}\text { Solution of } \\
\mathrm{NaN}_{3} \text {, } \\
\text { (concentration } \\
448 \mathrm{mM} \text { ), ml }\end{array}$ & $\begin{array}{c}\text { PBS, } \\
\mathrm{ml}\end{array}$ & $\begin{array}{l}\text { Complex of } \\
\text { biologically } \\
\text { active } \\
\text { substances, ml }\end{array}$ & $\begin{array}{l}\text { Solution of } \\
\mathrm{NaCl}, \\
(0.9 \%), \mathrm{ml}\end{array}$ \\
\hline Culture of negative control $\left(\mathrm{K}^{-}\right)$ & 12 & 0.5 & - & 0.5 & - & 1.0 \\
\hline Culture of positive control $\left(\mathrm{K}^{+}\right)$ & 12 & 0.5 & 0.5 & - & - & 1.0 \\
\hline Experimental cultures & 12 & 0.5 & 0.5 & - & 1.0 & - \\
\hline
\end{tabular}

Table 3. Incubation medium composition for determining SQR.

\begin{tabular}{ccc} 
Component & Quantity, ml & 8 \\
1/15 M solution of potassium dihydrogen phosphate & 32 & - \\
1/15 M solution of sodium dihydrogen phosphate & - & - \\
EDTA & - & - \\
sodium succinate dibasic hexahydrate & - & 340 \\
\hline
\end{tabular}


Table 4. Incubation medium composition for determining SQR.

\begin{tabular}{ccc}
\hline Component & Quantity, ml & Quantity, mg \\
\hline $1 / 15$ M solution of potassium dihydrogen phosphate & 8 & - \\
$1 / 15$ M solution of sodium dihydrogen phosphate & 32 & - \\
EDTA & - & 11 \\
p-nitrotetrazolium violet & - & 11 \\
$\alpha$-glycerol phosphate disodium salt hydrate & - & 630 \\
\hline
\end{tabular}

were washed with flowing water for 5 minutes and rinsed with distilled water. We carried out additional coloration of lymphocyte nuclei by $0.5 \%$ Janus green B solution for 5 seconds to relieve identification of the lymphocytes. Smears were washed with running water for 5 minutes and rinsed with distilled water. Smears were dried on the air. Cytochemical activity determination SQR and GPD2 based on the formation of formazan grains (purple granules) of p-nitrotetrazolium violet, which acts as a hydrogen acceptor from substrates, that fave been oxidated (sodium succinate dibasic hexahydrate for SQR and -glycerol phosphate disodium salt hydrate for GPD2). To determine the activity of SQR and GPD2 used cytochemical basic principle-the activity of these enzymes in the respective incubated cell is proportional to the square of formazan grains in it. Area of formazan grain was recorded using a light microscope AxioLab, A1 (Carl Zeiss, Germany) and camera AxioCam ERc5s (Carl Zeiss, Germany). Each smear was analyzed by 34 lymphocytes. These images are processed by a computer program UTHSCSA Image Tool, version 3.0 (The University of Texas Health Science Center, San Antonio, Texas, USA). In each lymphocyte area of formazan grains was measured. As a result, we received the sum of 34 cells from a smear in $\mathrm{mm}^{2}$. After this, we calculated the enzyme activity (SQR or GPD2), which numerically differed by the average area of formazan grains in one cell. Activity was expressed in $\mathrm{mm}^{2} / \mathrm{cell} / \mathrm{hour}$.

\subsection{Statistical Analysis}

To estimate statistical significance of changes in the experimental samples, in comparison to the control, as $\mathrm{K}^{+}$ and $\mathrm{K}^{-}$used nonparametric sign test $\mathrm{G}$. The critical level of significance for statistical criteria taken, so, that, equals $p<0.05[20]$.

\section{Results}

\subsection{Approaches to Aerobic Chain Energy Exchange Status Assessment}

One approach that provides the most objective picture of the state aerobic energy metabolism link are cytochemical methods of research activity of its enzymes in lymphocytes in vitro. They makes possible to evaluate the activity of enzymes in holistic cells, and mitochondrial enzymes, as in our case, in mitochondria, not destroying it. Because of this, cytochemical research techniques are highly sensitive, specific, informative, but most importantly the objectivity of the results [19]. That is why the cytochemical principle was chosen by us to study activity of one of the key enzymes in aerobic respiration-SQR and GPD2.

In the experiment, for modeling of disorders in energy metabolism, six hours after obtaining of primary lymphocytes, in experimental culture and culture $\mathrm{K}+\mathrm{NaN}_{3}$ (final concentration of $16 \mathrm{mM}$ ) was added. As is well known, this compound inhibits the complex IV work of the respiratory chain in mitochondria, which leads to dysfunction of the whole cell energy metabolism.

\subsection{SQR Activity Alteration in the Control and Experimental Lymphocytes' Culture}

In our research, at the initial stage of the experiment, after 18 hours of cultivation in the presence of $\mathrm{NaN}_{3}$, lymphocytes of the cultures $\mathrm{K}^{+}$(Figure 1) and experimental culture (Figure 2), in time of determining the activity of SQR, had a much smaller formazan grains area than the lymphocytes of culture $\mathrm{K}^{-}$(Figure 3). All this data indicates about significant decreased activity of SQR, both in the experimental culture, and the culture $\mathrm{K}^{+}$. As we can see in Figure 4 the average SQR activity in these cultures were 3.5 times less in comparison with the activity of lymphocytes SQR K ${ }^{-}$(0 hours of cultivation in the presence of the investigated complex of BAS).

To identify the effect of the investigated complex of BAS on the energy exchange, this complex was added to the experimental culture of lymphocytes that have been exposed $\mathrm{NaN}_{3}$. As a result, after only 4 hours of cultivation 


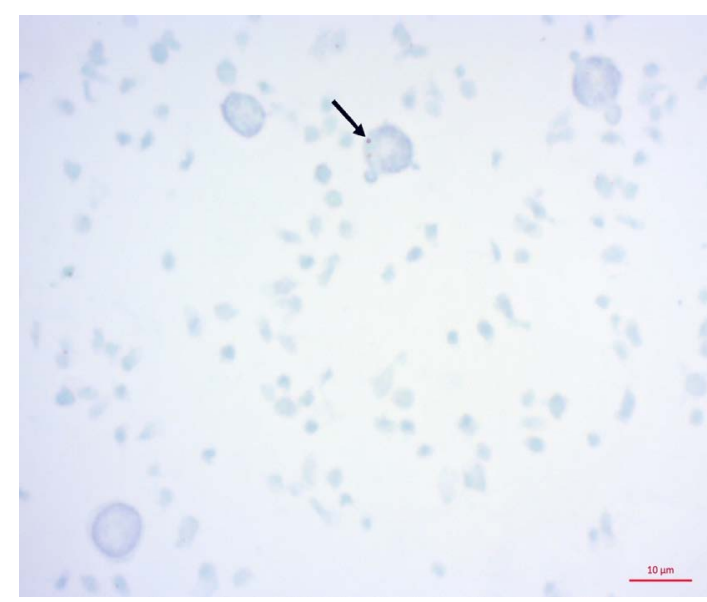

Figure 1. Cytochemical identification of SQR activity in lymphocyte (positive control), after 18 hours of $\mathrm{NaN}_{3}$ action. Example of a figure caption (figure caption).

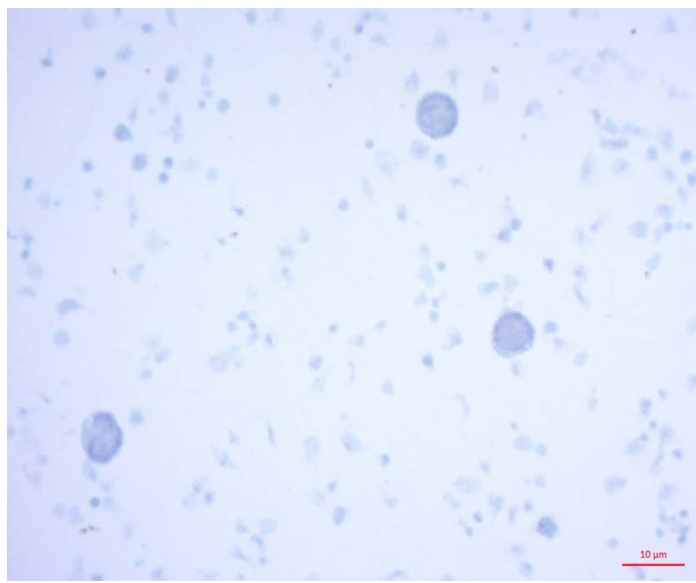

Figure 2. Cytochemical identification of SQR activity in lymphocytes (experimental culture) after 18 hours of $\mathrm{NaN}_{3}$ action before adding of the investigated complex of biologically active substances.

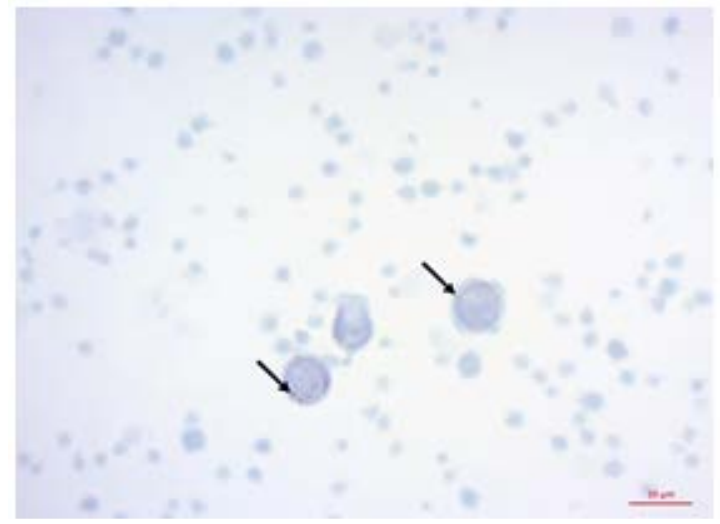

Figure 3. Cytochemical identification of SQR activity in lymphocyte culture (negative control) after 18 hours of PBS action on it. 
activity of SQR in experimental lymphocyte culture increased lymphocyte activity to a level $\mathrm{K}^{-}$, thus significantly exceeded the SQR activity of culture $\mathrm{K}^{+}$by 2.6-times (Figure 4). In Figure 5, we see that the area of the lymphocytes $\mathrm{K}^{-}$formazan granules and experimental cultures (Figure 6) is much larger than the area of these granules in cultures $\mathrm{K}^{+}$lymphocytes (Figure 7).

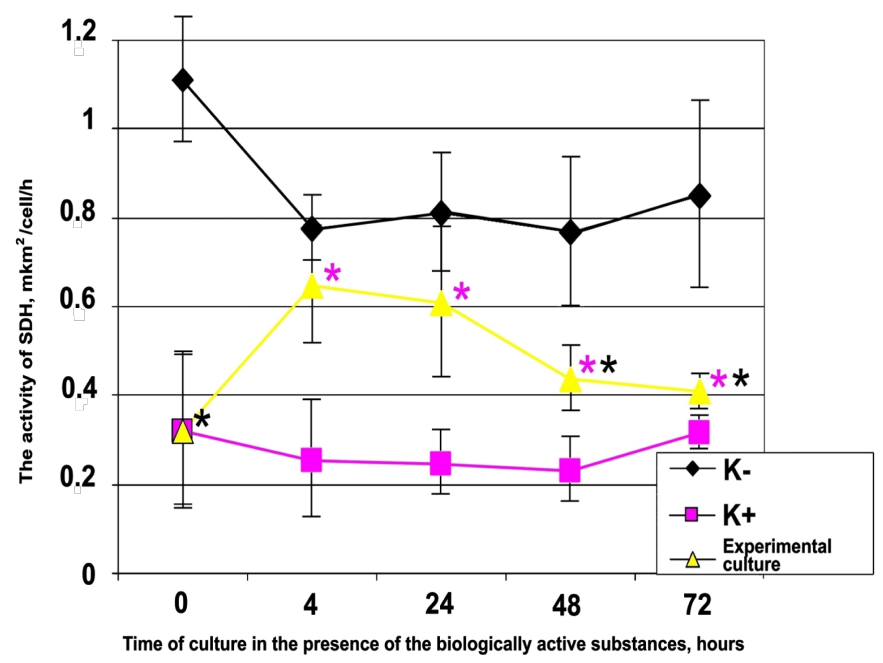

Figure 4. SQR activity of lymphocytes.

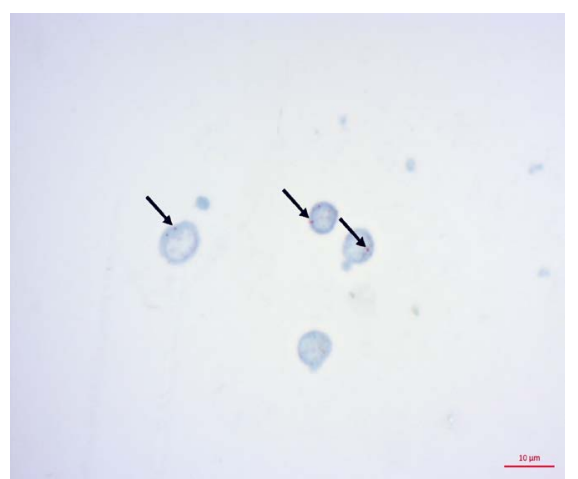

Figure 5. Cytochemical identification of SQR activity in lymphocyte culture (negative control) after 24 hours of PBS action and after 4 hours of $\mathrm{NaCl}$ action.

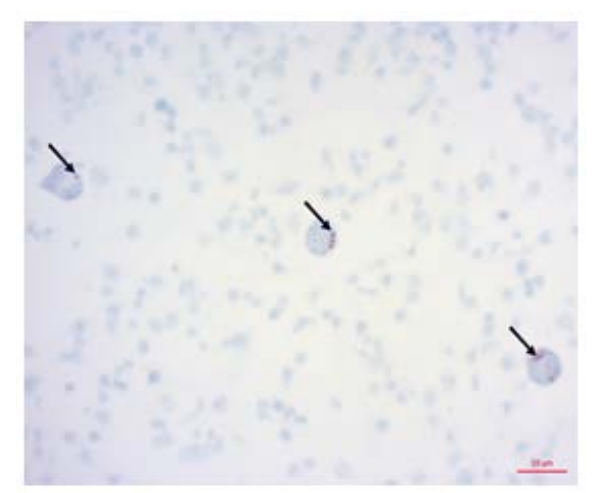

Figure 6. Cytochemical identification of SQR activity in lymphocytes (experimental culture) after 24 hours of $\mathrm{NaN}_{3}$ action, after 4 hours action of the complex of biologically active substances. 


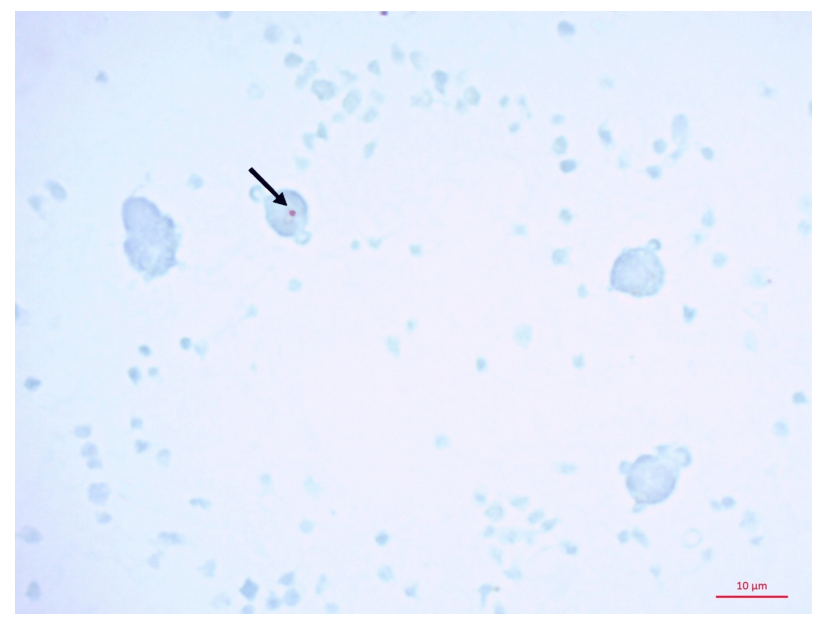

Figure 7. Cytochemical identification of SQR activity in lymphocyte culture (positive control) after 24 hours of $\mathrm{NaN}_{3}$ action and after 4 hours of action $\mathrm{NaCl}$.

A similar picture was also observed after 24-hour exposure to of the investigated complex of BAS. SQR activity of lymphocytes in the experimental samples was at the level of lymphocytes $\mathrm{K}^{-} \mathrm{SQR}$ activity, thus exceeding this index of cultures $\mathrm{K}^{+}$by 2.5 times. However, as seen in Figure 4, we found tendency to reduced activity of SQR in experimental culture of lymphocytes. Confirmation of this was the 48-hour exposure by the investigated complex of BAS to cells. At this stage of the experiment the activity of SQR lymphocyte in experimental culture was higher by 1.9 times than the corresponding figure in the energy metabolism of lymphocytes $\mathrm{K}^{+}$and was 1.7 times lower than the activity of SQR $\mathrm{K}^{-}$. The final stage of the experiment (72-hour exposure to a complex of BAS) was characterized by a further decreasing in the SQR activity of lymphocytes in experimental culture. It was only 1.3 times higher than the SQR activity of $\mathrm{K}^{+}$lymphocytes, but at the same time was 2.1 times lower than the SQR activity of lymphocytes $\mathrm{K}^{-}$(Figure 4).

\subsection{GPD2 Activity Alteration in the Control and Experimental Lymphocytes' Culture}

Changes in SQR activity were followed by no less significant dynamic activity of GPD2 Thus it is necessary to note that $\mathrm{NaN}_{3}$ can substantially activate mitochondrial GPD2. At the initial stage of the experiment, after 18 hours of cultivation in the presence of $\mathrm{NaN}_{3}$, lymphocytes cultures $\mathrm{K}^{+}$(Figure 8) and experimental culture (Figure 9), when determined the activity of GPD2, have a larger area of the formazan crystals than $\mathrm{K}^{-}$lymphocytes culture (Figure 10). This indicates about increasing of GPD2 activity, both in the experimental culture, and the culture of $\mathrm{K}^{+}$. In general, the activity of GPD2 in these lymphocyte cultures was higher by 2.2 times than the activity of GPD2 $\mathrm{K}^{-}$lymphocytes (Figure 11).

After adding into the experimental lymphocytes culture of the investigated BAS in ultra low concentrations, there was a significant inhibition of GPD2. As a result, after only 4 hours of cultivation, the activity of GPD2 lymphocytes in experimental culture decreased to the level of activity in lymphocytes $\mathrm{K}^{-}$and became lower by 1.6 times than activity of GPD2 culture $\mathrm{K}^{+}$(Figure 11). These changes are clearly visible in Figures 12-14.

Even more significant changes in the activity of GPD2 lymphocytes were identified at the end of 24 hour cultivation in the presence of ultra low concentrations of the experimental BAS. At this stage of the experiment, the activity of GPD2 lymphocytes experimental culture was 2.2 times lower than in cultures $\mathrm{K}^{+}$(Figure 11). The tendency at reduced activity of GPD2 lymphocytes in experimental culture relatively the culture of $\mathrm{K}^{+}$remained and after 48-hour cultivation with experimental complex of BAS. The activity of lymphocytes GPD2 in experimental culture was less than the corresponding index in $\mathrm{K}^{+}$lymphocytes by 2.3 times, and in fact as active as GPD2 in $\mathrm{K}^{-}$(Figure 11). 72-hour joint action on lymphocytes by ultra low concentrations of BAS has caused at least a significant reduction in GPD2 activity of the experimental samples than the previous three stages of the experiment. Thus the activity of lymphocytes GPD2 experimental culture was only 1.3 times lower than in samples $\mathrm{K}^{+}$while not statistically different from the activity of lymphocytes culture GPD2 $\mathrm{K}^{-}$(Figure 11). 


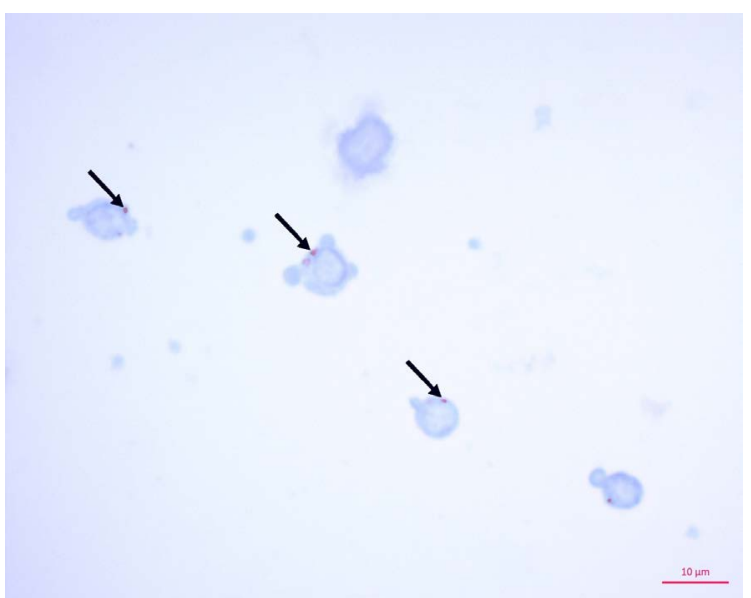

Figure 8. Cytochemical identification of GPD2 activity in lymphocyte culture (positive control), after 18 hours of NaN3 action on it.

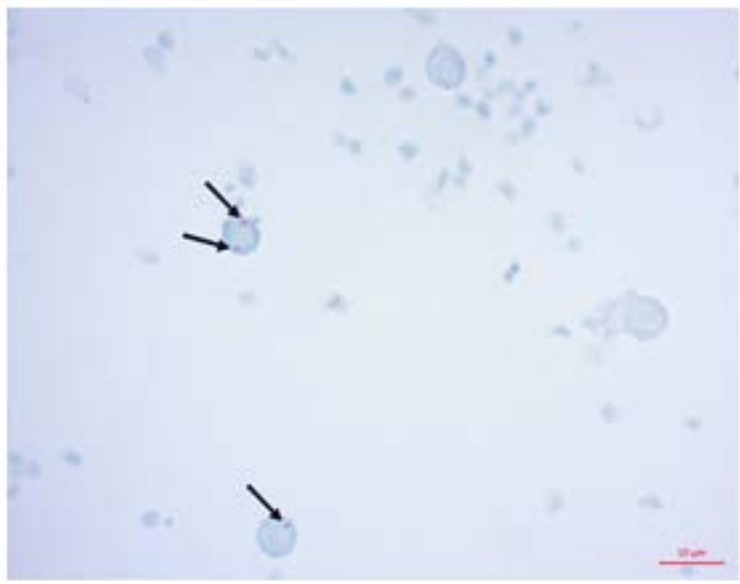

Figure 9. Cytochemical identification of GPD2 activity in lymphocytes (experimental culture) after 18 hours of $\mathrm{NaN}_{3}$ action, before adding of the investigated complex of biologically active substances.

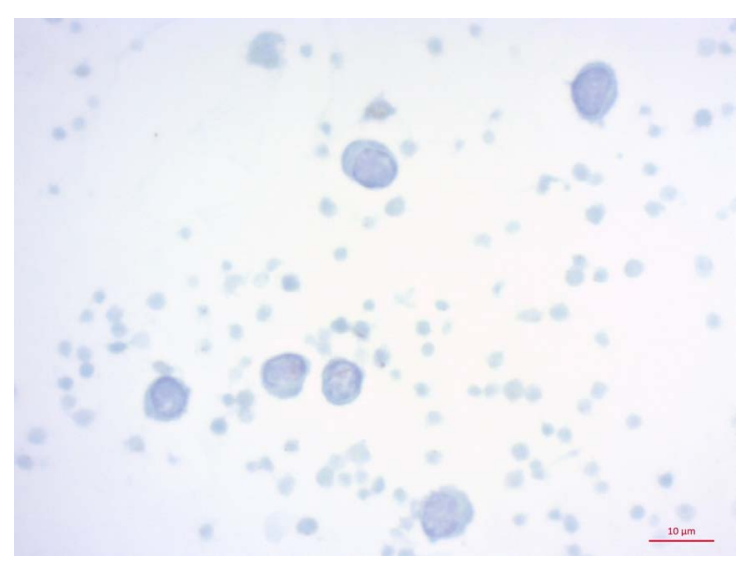

Figure 10. Cytochemical identification of GPD2 activity in lymphocyte culture (negative control), after 18 hours of PBS action on it. 


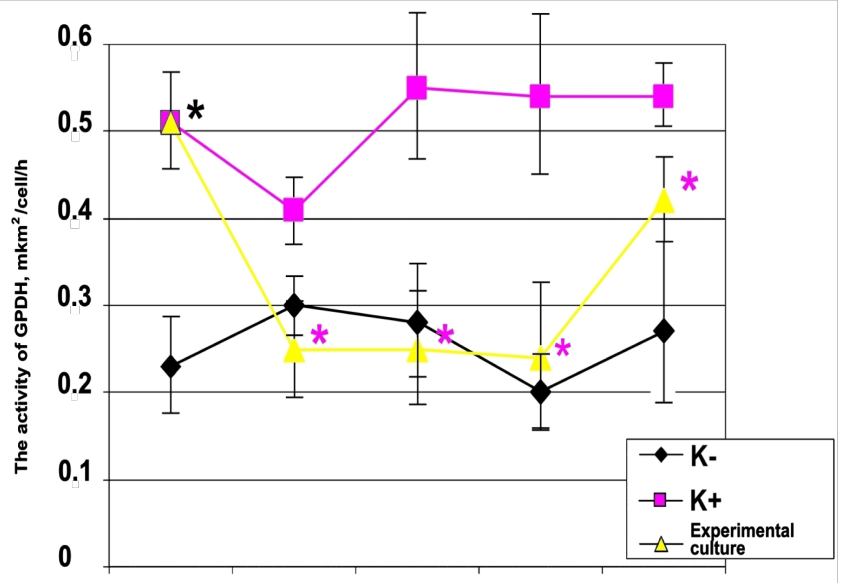

Figure 11. GPD2 activity of lymphocytes.

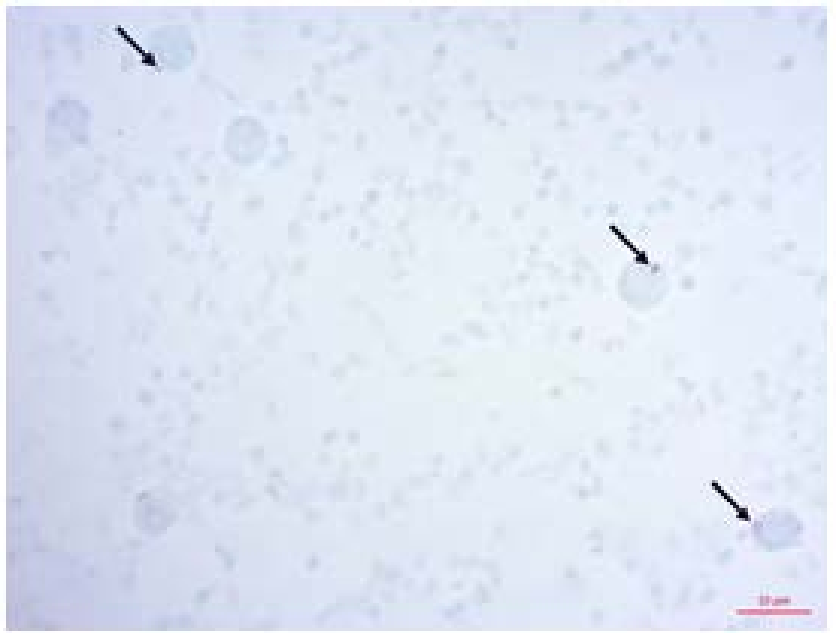

Figure 12. Cytochemical identification of GPD2 activity in lymphocyte culture (positive control) after 24 hours of $\mathrm{NaN}_{3}$ action on it, after 4 hours of $\mathrm{NaCl}$ action.

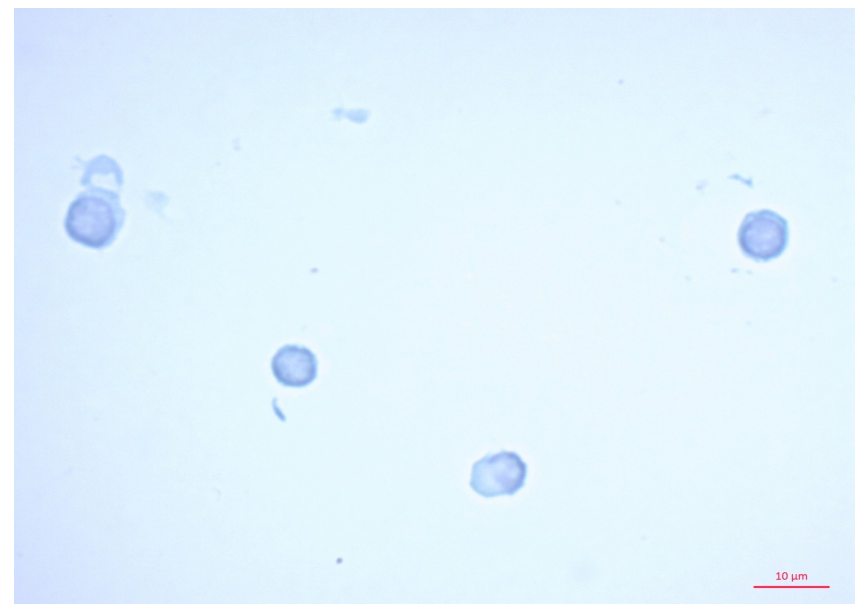

Figure 13. Cytochemical identification of GPD2 activity in lymphocyte (experimental culture) after 24 hours of $\mathrm{NaN}_{3}$ action on it, after 4 hours of complex of biologically active substances action. 


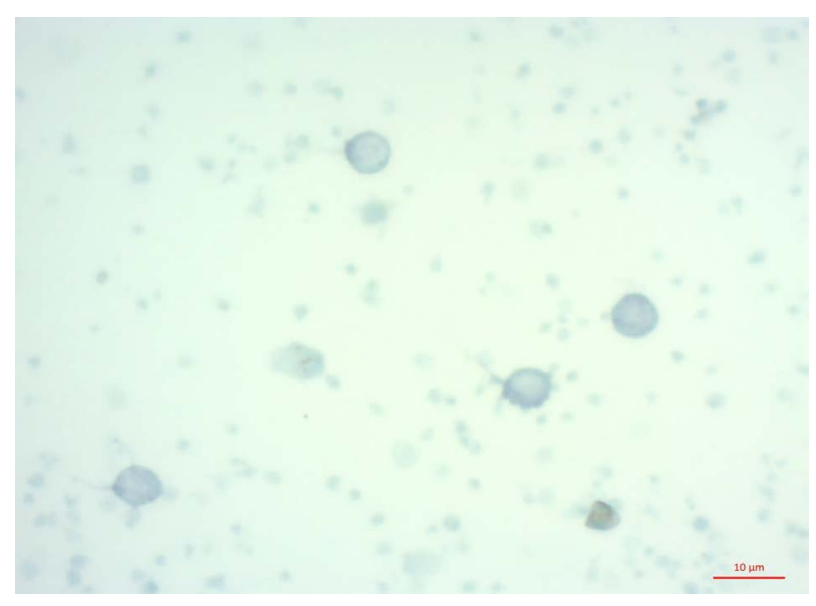

Figure 14. Cytochemical identification of GPD2 activity in lymphocyte culture (negative control) after 24 hours of PBS action on it, after 4 hours of $\mathrm{NaCl}$ action.

\section{Discussion}

\subsection{Certain Aspects of SDH and GPDH Functioning}

As it is known, SQR and GPD2 catalyze some of the most important reactions of aerobic energy metabolism link. Thus SQR in the citric acid cycle catalyzes the reversible oxidation of succinic acid to fumaric acid. The electrons are transferred from the SQR directly to the respiratory chain complex II, and then on coenzyme Q, which makes SQR a major enzymes of energy exchange. Mitochondrial GPD2 is the main component of the glycerophosphate shuttle transfer mechanism of recovered equivalents from cytosol NADH to the mitochondrial FAD [7].

\subsection{Compensatory-Adaptive Response of SDH and GPDH to the $\mathrm{NaN}_{3}$ Influence}

Conducted researches have allowed to reveal significant change in the activity of SQR and GPD2. At the initial stage of the experiment, the inhibitory effect of $\mathrm{NaN}_{3}$ on aerobic respiration was realized through substantial reduction of SQR activity with parallel activation of GPD2. These changes were observed in the $\mathrm{K}^{+}$culture and experimental culture after 18-hour cultivation with $\mathrm{NaN}_{3}$, before entering to the last of the experimental BAS. Of course, such changes in the activity of enzymes in aerobic respiration were adaptive-compensatory response. Reduced SQR activity in response to the impaired function of cytochrome C oxidase by azide undoubtedly wore a compensatory character. It is known that the normal functioning of all the components of the respiratory chain cannot be performed unless it is accompanied by phosphorylation of ADP. That is why, it is logical to inhibit SQR in response to an impaired function of cytochrome $\mathrm{C}$ oxidase in the initial stage of the experiment (Figure 4). 0 hours of cultivation in the presence of the investigated complex of BAS. At the same time lack of ATP synthesis in these conditions led to incorporation of cellular adaptation mechanisms-activate job glycerophosphate shunt, which was aimed on creating of the gradient of restored FAD, enough for resuming of the normal respiratory chain functioning. This was expressed in the activation of mitochondrial function GPD2 (Figure 11). 0 hours of cultivation in the presence of the investigated complex of BAS.

\subsection{The Effect of Ultra Low Concentrations of BAS on Activity of SQR and GPD2}

Adding to the experimental lymphocyte culture of the investigated complex BAS and their subsequent cultivation for 4 hours led to significant changes in the activity of SQR and GPD2. Thus in the experimental lymphocyte culture has been rapid increase of SQR activity on the background of simultaneous inhibition of GPD2 (Figure 4 and Figure 11). It is important to note that the activity of these enzymes in experimental culture were at the level of relevant indicators of culture $\mathrm{K}^{-}$. This may indicate only one thing-4 hours of cultivating experimental lymphocyte culture with the investigated complex of BAS stabilize the processes of aerobic respiration in it to the normal level. Parameters of functioning of the second complex in respiratory chain and glycerophos- 
phate shuttling mechanism in the experimental culture corresponded to the index $\mathrm{K}^{-}$culture, which was not exposed by $\mathrm{NaN}_{3}$, and investigated complex of BAS.

This pattern was preserved in the next phase of the experiment. 24-hour of experimental lymphocyte culture cultivation with the investigated complex of BAS has led to stabilize SQR and GPD2 activity to the level $\mathrm{K}^{-}$. At the same time, in spite of significant excess activity of SQR in experimental culture, SQR activity in the culture $\mathrm{K}^{+}$had tendency for the relative decline in the first (Figure 4). At the same time, we didn't observed significant increase in GPD2 activity. In contrast, in comparison with the preceding stage of the experiment, it has decreased relative to the GPD2 activity culture $\mathrm{K}^{+}$(Figure 11). In this case, we can state the fact, that the investigated BAS used in ultra low concentrations is able in 24 hours effectively stabilize the aerobic link of energy exchange which has undergone the action of $\mathrm{NaN}_{3}$.

Several other changes SQR activity was identified by us in the 48-hour action on experimental lymphocytes culture of the investigated complex BAS used in ultra low concentrations. Although, as in the previous stage of the experiment, the activity of SQR experimental culture exceeded that index in culture $\mathrm{K}^{+}$, but it was significantly lower than SQR activity of culture $\mathrm{K}^{-}$(Figure 4). This may indicate a gradually decreased effect of investigated BAS in the complex II of the mitochondria respiratory chain. At the same time, low activity glycerophosphate shunt indicates about significant effect of the BAS complex on the link of intracellular aerobic respiration. Activity of GPD2 in experimental culture was at the level $\mathrm{K}^{-}$culture thus was significantly lower than in culture to $\mathrm{K}^{+}$(Figure 11). All this indicates about significant energotropic effect of ultra low concentrations of the experimental BAS in the complex 48-hour operation.

At the final stage of the experiment (72-hour incubation of experimental culture with a complex of BAS) observed similar changes in the activity of the investigated enzymes, as in the previous stage. SQR activity in experimental cultures exceeded this index in culture $\mathrm{K}+$, but at the same time the SQR activity was lower than in culture $\mathrm{K}^{-}$(Figure 4). On this background activity of GPD2 in experimental culture was lower than in cultures $\mathrm{K}^{+}$(Figure 11). All this indicates about continuing effect of ultra low concentrations of the experimental BAS on the intracellular aerobic respiration link.

\section{Conclusions}

So carried out researches have allowed to reveal the growth of SQR activity in the experimental lymphocyte culture relatively culture $\mathrm{K}^{+}$during the entire experiment. At the same time, activity of the main of enzyme glycerophosphate shuttle mechanism-mitochondrial GPD2 in the experimental lymphocytes culture is significantly lower relative to the corresponding index in $\mathrm{K}^{+}$culture. All of this suggests powerful energotropic effect of ultra low concentrations of experimental BAS in the lymphocyte aerobic element of energy exchange. It is also important to note relative decreasing of this effect starting from 48-hour incubation of experimental culture with the complex of BAS. This is evidenced by decreased activity of SQR in the experimental lymphocyte culture, relative to the corresponding index culture $\mathrm{K}^{-}$.

So we have found that extremely low concentrations of selected BAS with their complex impact on human lymphocytes in vitro, can effectively neutralize the inhibitory effect of $\mathrm{NaN}_{3}$ on processes of aerobic energy metabolism link. Certainly, a hard research work should be done for understanding the mechanism of ultra low doses action of different substances and the chosen BAS complex in particular. We hope that our research will shed light on solving the fundamental question, namely how such ultra low doses substances can lead to significant biochemical, physiological, immunologic and other effects.

\section{References}

[1] Sukhorukov, V.S. and Nikolaeva, E.A. (2004) Violation of the Cellular Energy Exchange in Children. Ataes Medica Soft, Moscow, 79.

[2] Copeland, W. (2002) Mitochondrial DNA Methods and Protocols. Methods in Molecular Biology, $197,401$.

[3] Weissig, V. and Edeas, M. (2015) Mitochondrial Medicine. Methods in Molecular Biology. Springer Science + Business Media, New York.

[4] Gvozdjáková, A. (2008) Mitochondrial Medicine. Mitochondrial Metabolism, Diseases, Diagnosis and Therapy. Springer Science + Business Media, B.V. Dordrecht, Netherlands.

[5] Koene, S. and Smeitink, J. (2011) Mitochondrial Medicine. Journal of Inherited Metabolic Disease, 34, 247-248. http://dx.doi.org/10.1007/s10545-011-9292-x 
[6] Dorey, E. (2014) Mitochondrial Medicine. Nature Biotechnology, 32, 300. http://dx.doi.org/10.1038/nbt0414-300a

[7] Sukhorukov, V.S. (2011) Studies of Mitochondrial Pathology. Publishing House “Medpraktika-M”, Moscow, 288.

[8] Scatena, R. (2012) Mitochondria and Drugs. Advances in Experimental Medicine and Biology, 942, 329-346. http://dx.doi.org/10.1007/978-94-007-2869-1_15

[9] Parikh, S., Saneto, R., Falk, M., Anselm, I., Cohen, B. and Haas, R. (2009) A Modern Approach to the Treatment of Mitochondrial Disease. Current Treatment Options in Neurology, 11, 414-430. http://dx.doi.org/10.1007/s11940-009-0046-0

[10] Andreazza, A. and Scola, G. (2015) Toxicology Studies-Cells, Drugs and Environment. In: Andreazza, A. and Scola, G., Eds., InTech, Rijeka, 62-63. http://dx.doi.org/10.5772/58714

[11] Khuda-Bukhsh, A.R. (2014) Current Trends in High Dilution Research with Particular Reference to Gene Regulatory Hypothesis. The Nucleus, 57, 1, 3-17. http://dx.doi.org/10.1007/s13237-014-0105-0

[12] Wälchli, C., Baumgartner, S. and Bastide, M. (2006) Effect of Low Doses and High Homeopathic Potencies in Normal and Cancerous Human Lymphocytes: An in Vitro Isopathic Study. The Journal of Alternative and Complementary Medicine, 12, 421-427.

[13] Lenger, K., Bajpai, R. and Spielmann, M. (2014) Identification of Unknown Homeopathic Remedies by Delayed Luminescence. Cell Biochemistry and Biophysics, 68, 321-334. http://dx.doi.org/10.1007/s12013-013-9712-7

[14] Ezz, H. and Elkala, S. (2015) Ultra-Low-Dose Naloxone Added to Fentanyl and Lidocaine for Peribulbar Anesthesia: A Randomized Controlled Trial. Egyptian Journal of Anesthesia, 31, 161-165. http://dx.doi.org/10.1016/j.egja.2014.12.005

[15] Yovell, Y., Bar, G., Mashiah, M., Baruch, Y. and Briskman, I. (2015) Ultra-Low-Dose Buprenorphine as a Time-Limited Treatment for Severe Suicidal Ideation: A Randomized Controlled Trial. The American Journal of Psychiatry, 15, 231-241 http://dx.doi.org/10.1176/appi.ajp.2015.15040535

[16] Thompson, T. and Weiss, M. (2006) Homeopathy-What Are the Active Ingredients? An Exploratory Study Using the UK Medical Research Council's Framework for the Evaluation of Complex Interventions. BMC Complementary and Alternative Medicine, 12, 6-37. http://dx.doi.org/10.1186/1472-6882-6-37

[17] Winchester, R.J. and Ross, G. (1976) Methods for Enumerating Lymphocyte Populations in Manual of Clinical Immunology. In: Rose, N.R. and Friedman, H., Eds., American Society for Microbiology, Washington DC, 64-76.

[18] Burlakova, E.B., Conrad, A.A. and Maltseva, E.L. (2003) Ultralow Doses Action of Biologically Active Substances and Low-Intensity Physical Factors. Chemical Physics, 22, 21-40.

[19] Yagoda, A.V. and Lokteva, N.A. (2005) Clinical Cytochemistry. SiGMA, Stavropol, 485.

[20] Lapach, S.N., Chubenko, A.V. and Babich, P.N. (2001) Statistical Methods in Biomedical Research Using Excel. 2nd Edition, MORION, Kyiv, 196. 